\title{
Improving pointing of Torun 32-m radio telescope: effects of rail surface irregularities
}

\author{
Bartosz Lew ${ }^{1}$ (D)
}

Received: 28 July 2017 / Accepted: 17 January 2018 / Published online: 7 February 2018

(C) The Author(s) 2018. This article is an open access publication

\begin{abstract}
Over the last few years a number of software and hardware improvements have been implemented to the 32-m Cassegrain radio telescope located near Torun. The 19-bit angle encoders have been upgraded to 29-bit in azimuth and elevation axes. The control system has been substantially improved, in order to account for a number of previously-neglected, astrometric effects that are relevant for milli-degree pointing. In the summer 2015, as a result of maintenance works, the orientation of the secondary mirror has been slightly altered, which resulted in worsening of the pointing precision, much below the nominal telescope capabilities. In preparation for observations at the highest available frequency of $30-\mathrm{GHz}$, we use One Centimeter Receiver Array (OCRA), to take the most accurate pointing data ever collected with the telescope, and we analyze it in order to improve the pointing precision. We introduce a new generalized pointing model that, for the first time, accounts for the rail irregularities, and we show that the telescope can have root mean square pointing accuracy at the level $<8^{\prime \prime}$ and $<12^{\prime \prime}$ in azimuth and elevation respectively. Finally, we discuss the implemented pointing improvements in the light of effects that may influence their long-term stability.
\end{abstract}

Keywords Astronomical instrumentation - Methods and techniques · Telescopes · Radio continuum: general $\cdot$ Methods: observational

Bartosz Lew

bartosz.lew@umk.pl

1 Centre for Astronomy, Faculty of Physics, Astronomy and Informatics,

Nicolaus Copernicus University, Grudziadzka 5, 87-100 Torun, Poland 


\section{Introduction}

The Torun 32-meter radio telescope is a classical Cassegrain telescope with altazimuth, wheel-on-track mounting. Located in central Europe, and operated 24-h a day, it is one of the European VLBI Network (EVN) nodes, capable of observing at frequencies from $\sim 1 \mathrm{GHz}$ to $\sim 30 \mathrm{GHz}$ in continuum and spectroscopic modes at the selected bands.

Over the last several months a few important astrometric improvements have been introduced into the control system. More improvements were implemented over the period of last few years, including weather dependent radio refraction and corrections due to differences between the Coordinated Universal Time (UTC) and Universal Time (UT1). The secondary mirror of the Cassegrain system by design has five degrees of freedom: 2 for lateral translations in the focal plane, 1 for translations along the optical axis, and 2 for rotations about secondary focus. Given that the mirror drives become unreliable over time in 2015 the mirror has been fixed. As a result, its orientation and gravitational sag have changed. Furthermore, the 19-bit angle encoders have been upgraded in azimuth and elevation axes in 2013 and 2014 respectively, and now provide position information with sub-arcsecond resolution.

For about ten years, since the initial fixation of the secondary mirror in 2006, and since introducing the pointing model used for the observations presented in this work, it has been known that the residuals between the measured pointing corrections, and the best-fitting pointing model, exhibit patterns that vary faster in angular space than the model can accommodate. However, only with the advent of higher precision 22$\mathrm{GHz}$ spectral pointing observations, and the data acquired in the continuum with OCRA-p (One Centimeter Receiver Array prototype) radiometer [6], and by using an improved version of the control system, which provides position readouts at higher frequency, and also by improving the extraction algorithms for position corrections from cross-scan observations, in the year 2015, it become possible to undoubtedly associate these high frequency patterns to irregularities of the rail as the telescope trolleys roll over the welding points. All of these observations require introducing a more general pointing model and its calibration by means of the new pointing data. In preparation for the observation programs carried out at frequencies above $20 \mathrm{GHz}$ $[9,18-20,23,25,32]$, we carry out pointing campaigns aiming at improving the pointing precision to one-tenth of the highest frequency beam, i.e. $\sim 0.002^{\circ}$.

In this work we introduce a generalized pointing model, implement it into the control system and discuss the resulting improvements. The assumed target precision, the order of one milli-degree, may also require precise thermal control of the supporting structure since seasonal, day to night and sun to shade temperature variations may have significant effects on pointing capabilities of large-aperture and/or millimeterwave telescopes [1-3, 12, 24, 26, 27, 31, 33, 36]. Other effects such as strong wind can also impact surface deformation and pointing at the level of several seconds of arc [34, 35] and lead to significant loses of available observing time [28, 29].

It is known that the surface accuracy of the rail, its design and component connections may also significantly impact pointing capabilities of large telescopes [8, $15,17,22,30]$. While controlling parameters such as temperature or inclination of the structure may also be critical for maintaining high pointing performance, in the 
present work on the 32-m Torun radio telescope, we primarily focus on modeling the previously-neglected effects associated with the telescope rail, and how they limit pointing capabilities, while we leave discussing thermal effects to a separate analysis.

The impact of the rail surface irregularity on the orientation of the antenna, and consequently on the amplitude of the associated position corrections can be accurately modeled using data obtained from inclinometers installed on the structure of the telescope (e.g. [10, 11]). In the absence of the inclinometers, an alternative approach is possible, which is to extract the position corrections directly from pointing observations.

In Section 2 we lay out the notation and basic relations used throughout the rest of the paper. In Section 3 we describe pointing models used with the 32-m Torun radio telescope. Pointing observations and data processing are discussed in Sections 4 and 5 respectively. The main results are presented in Sections 6 and 7 where we calibrate the extended pointing models and present the impact of rail irregularities. We discuss the results in connection with independent measurements of the rail in Section 9 and then conclude in Section 10.

\section{Coordinates and reference frames}

When an observer demands to track a distant source at equatorial coordinates $\left(\alpha_{e}, \delta_{e}\right)$ for a given epoch (e.g. J2000), these coordinates are converted to $\left(\alpha_{S}, \delta_{S}\right)$ coordinates of the current date, accounting for precession, nutation, and annual aberration. The corresponding horizontal celestial coordinates $\left(A_{S}, Z_{S}\right)$ of the date are calculated using standard spherical trigonometry rotations for the actual local mean sidereal time. These coordinates are referred to as "SET" coordinates and they represent the actual, in-vacuum direction towards the source in horizontal coordinates.

In order to track the source, the control system needs to minimize the difference between the updated $\left(A_{S}, Z_{S}\right)$ direction and the "TRUE" (in-vacuum) direction of the selected telescope beam $\left(A_{T}, Z_{T}\right)$, accounting for its offset $\left(B_{A}, B_{Z}\right)$ from the telescope optical axis. The corresponding $\left(\alpha_{T}, \delta_{T}\right)$ are calculated and updated using $\left(A_{T}, Z_{T}\right)$ for the actual time. When tracking, the horizontal coordinates of the optical axis are related to the "TRUE" coordinates of the beam through:

$$
\begin{aligned}
& A_{T}=A_{\mathrm{oa}}-O_{A}-B_{A} / \sin \left(Z_{\mathrm{oa}}\right) \\
& Z_{T}=Z_{\mathrm{oa}}-O_{Z}-B_{Z}+R,
\end{aligned}
$$

where $R$ is a refraction angle discussed latter and $O_{A}$ and $O_{Z}$ are azimuth and zenith distance offsets which are added if requested.

The condition for tracking a source at fixed offset defined in azimuth-zenithdistance space is:

$$
\begin{aligned}
& A_{S}+O_{A}=A_{T} \\
& Z_{S}+O_{Z}=Z_{T} .
\end{aligned}
$$

For the case when the receiver beam tracks the source we have: $O_{A}=0$ and $O_{Z}=$ 0 . In the version of the control system which has been used to gather the pointing data 
presented in this work, the receiver beam offset $B_{A}$ scales with the zenith distance as $B_{A} / \sin \left(Z_{\mathrm{oa}}\right)(1 \mathrm{a}, \mathrm{b})$. We will hereafter refer to this version as fast_track .

While tracking, these coordinates are sensitive to switching from one off-axis receiver to another, as the tracking telescope must physically slightly reorient itself when a new offset receiver (and therefore offset beam) is requested. At the same time the "SET" coordinates are, obviously, not sensitive to these changes. The pair of "TRUE" and "SET" coordinates is provided numerically to other programs and to users via local network. While tracking a source, "TRUE" and "SET" coordinates are made equal to within tracking precision by a proportional-integral-derivative (PID) controller.

For each moment in time the position encoders read the actual orientation of the telescope in its own coordinate system, that only crudely approximates the horizontal coordinate system. We will call these coordinates $A_{E}$ and $Z_{E}$. In order to relate these coordinates to the "TRUE" (in-space) coordinates $\left(A_{T}, Z_{T}\right)$ when the telescope is tracking a source, they need to be corrected for (i) tilt of the vertical axis with respect to geodetic zenith, (ii) skew of the elevation axis, (iii) focus box offsets (iv) gravitational sag of the Cassegrain mirror (v) rail deficiencies (vi) and other effects resulting from construction defects that cannot be easily modeled. Hereafter, all of these corrections are jointly called COR, and they allow us to connect the coordinates of the telescope own coordinate system to the horizontal, in-space coordinates of the selected beam via the weather-dependent atmospheric refraction angle $R>0$ :

$$
\begin{aligned}
A_{T}+O_{A}+B_{A} / \sin \left(Z_{\mathrm{oa}}\right) & =A_{E}-C_{A}\left(A_{E}, Z_{E}\right)-T_{A}\left(A_{E}, Z_{E}\right) \\
Z_{T}+O_{Z}+B_{Z} & =Z_{E}-C_{Z}\left(A_{E}, Z_{E}\right)-T_{Z}\left(A_{E}, Z_{E}\right)+R\left(Z_{\mathrm{oa}}, T, P, H\right),
\end{aligned}
$$

where $R$ is a function of $Z_{\mathrm{oa}}$ (zenith distance of the optical axis in the horizontal coordinate system) and actual temperature $(T)$, pressure $(P)$ and humidity $(H)$, provided to the control system from local meteorological station. The control system also accommodates for additional position corrections $T_{A}$ and $T_{Z}$ which are taken from look-up tables, although these were not used during the pointing campaign i.e. for the data considered in this work $\left(T_{A}, T_{Z}\right)=(0,0)$. Obviously, the pointing data discussed in Section 5, have no user-defined offsets, hence in this analysis $\left(O_{A}, O_{Z}\right)=(0,0)$. The $C_{A}$ and $C_{Z}$ terms in $(3 \mathrm{a}, \mathrm{b})$ represent all of the COR effects. In this setting, pointing imperfections result mainly from our ignorance about the true orientation of the optical axis. In the case when COR is not exact, pointing measurements will indicate non-zero position corrections at the source direction. We can account for that by rewriting $(3 a, b)$ as:

$$
\begin{aligned}
A_{T}+B_{A} / \sin \left(Z_{\mathrm{oa}}\right)+\Delta_{A} & =A_{E}-C_{A}\left(A_{E}, Z_{E}\right) \\
Z_{T}+B_{Z}+\Delta_{Z} & =Z_{E}-C_{Z}\left(A_{E}, Z_{E}\right)+R\left(Z_{\mathrm{oa}}, T, P, H\right),
\end{aligned}
$$

where $\left(\Delta_{A}, \Delta_{Z}\right)$ are the measured position corrections. Using (1a,b) this can be rewritten in terms of the coordinates of the telescope optical axis:

$$
\begin{aligned}
& A_{\mathrm{oa}}+\Delta_{A}=A_{E}-C_{A}\left(A_{E}, Z_{E}\right) \\
& Z_{\mathrm{oa}}+\Delta_{Z}=Z_{E}-C_{Z}\left(A_{E}, Z_{E}\right) .
\end{aligned}
$$


Table 1 summarizes the coordinate systems and naming conventions introduced in this section.

\section{Pointing model}

In the fast_track version of the control system the position corrections (COR) are defined by an analytic pointing model called "Model 4c" [4, 5] which is based on the model derived by [13]. This model was used during the pointing observations (Section 4).

The problem of improving the 32-m Torun radio telescope pointing precision is a matter of finding new analytic models as a replacement for $C_{A}\left(A_{E}, Z_{E}\right)$ and $C_{Z}\left(A_{E}, Z_{E}\right)$ functions $(5 \mathrm{a}, \mathrm{b})$ such that they minimize the position corrections

Table 1 List of coordinates and naming conventions

\begin{tabular}{|c|c|c|}
\hline Control system name & Name & Comment \\
\hline SET & $\left(\alpha_{S}, \delta_{S}\right)$ & $\begin{array}{l}\text { Equatorial coordinates of the selected source, calculated from } \\
\text { user-provided coordinates for a given epoch }\end{array}$ \\
\hline SET & $\left(A_{S}, Z_{S}\right)$ & $\begin{array}{l}\text { Actual (in-vacuum) horizontal coordinates of the selected source, } \\
\text { corresponding to }\left(\alpha_{S}, \delta_{S}\right) \text { at the actual UT1 time }\end{array}$ \\
\hline TRUE & $\left(A_{T}, Z_{T}\right)$ & Actual (in-vacuum) horizontal coordinates of the receiver beam \\
\hline \multirow[t]{9}{*}{ TRUE } & $\left(\alpha_{T}, \delta_{T}\right)$ & $\begin{array}{l}\text { Equatorial coordinates coordinates of the receiver beam corres- } \\
\text { ponding to }\left(A_{T}, Z_{T}\right)\end{array}$ \\
\hline & $\left(A_{\mathrm{oa}}, Z_{\mathrm{oa}}\right)$ & $\begin{array}{l}\text { Horizontal coordinates of the telescope optical axis. These coor- } \\
\text { dinates are calculated by the control system from }\left(A_{E}, Z_{E}\right) \text { with } \\
\text { assumptions on pointing model (COR). } A_{\mathrm{oa}}=A_{E}-C_{A}\left(A_{E}, Z_{E}\right) \\
\text { and } Z_{\mathrm{oa}}=Z_{E}-C_{Z}\left(A_{E}, Z_{E}\right) \text {. }\end{array}$ \\
\hline & $\left(A_{E}, Z_{E}\right)$ & $\begin{array}{l}\text { Coordinates of the optical axis in the telescope own coordi- } \\
\text { nate system that approximates horizontal coordinate system with } \\
\text { imperfections described by COR (see Section 2). These coordi- } \\
\text { nates are read, but not available to the user. }\end{array}$ \\
\hline & $\left(C_{A}, C_{Z}\right)$ & $\begin{array}{l}\text { pointing model corrections }(\mathrm{COR}) \text { in } A_{E} \text { azimuth and } Z_{E} \text { zenith } \\
\text { distance }\end{array}$ \\
\hline & $\left(T_{A}, T_{Z}\right)$ & Look-up table corrections in $A_{E}$ azimuth and $Z_{E}$ zenith distance \\
\hline & $R$ & Weather dependent radio refraction angle. $R>0$ \\
\hline & $\left(O_{A}, O_{Z}\right)$ & $\begin{array}{l}\text { User-defined position offset, defined in the horizontal coordinate } \\
\text { system. } O_{A} \text { increases westwards. } O_{Z} \text { increases downwards. }\end{array}$ \\
\hline & $\left(B_{A}, B_{Z}\right)$ & $\begin{array}{l}\text { Receiver beam offset from optical axis defined in the hori- } \\
\text { zontal coordinate system. } B_{A} \text { depends on elevation as } B_{A}= \\
B_{A}\left(Z_{\mathrm{oa}}=90^{\circ}\right) / \sin \left(Z_{\mathrm{oa}}\right) . B_{A} \text { increases westwards. } B_{Z} \text { increases } \\
\text { downwards. }\end{array}$ \\
\hline & $\left(\Delta_{A}, \Delta_{Z}\right)$ & $\begin{array}{l}\text { Position corrections measured using the fast_track version } \\
\text { of the control system that uses the "Model } 4 \mathrm{c} \text { " pointing correc- } \\
\text { tions model (see Section } 3 \text { ). These position corrections need to } \\
\text { be applied at the top of the used pointing model (COR) to point } \\
\text { a beam at the requested source. }\end{array}$ \\
\hline
\end{tabular}


$\left(\Delta_{A}, \Delta_{Z}\right)$ amended by the pointing model used to measure them. For this purpose we define:

$$
\begin{aligned}
& \epsilon_{A}\left(A_{E}, Z_{E}\right)=\Delta A+C_{A}\left(A_{E}, Z_{E}\right) \\
& \epsilon_{Z}\left(A_{E}, Z_{E}\right)=\Delta Z+C_{Z}\left(A_{E}, Z_{E}\right) .
\end{aligned}
$$

We also define a new pointing corrections model for azimuth $M_{A}\left(\mathbf{p}, A_{E}, Z_{E}\right)$ and zenith distance $M_{Z}\left(\mathbf{q}, A_{E}, Z_{E}\right)$ and we fit pointing measurements by minimizing:

$$
\begin{aligned}
& \chi_{A}^{2}=\sum_{i=1}^{N} \frac{w_{i}^{2}}{\sigma_{A_{i}}^{2}}\left(\epsilon_{A_{i}}-M_{A}^{(Y)}\left(\mathbf{p}, A_{E}, Z_{E}\right)\right)^{2} \\
& \chi_{Z}^{2}=\sum_{i=1}^{N} \frac{w_{i}^{2}}{\sigma_{Z_{i}}^{2}}\left(\epsilon_{Z_{i}}-M_{Z}^{(Y)}\left(\mathbf{q}, A_{E}, Z_{E}\right)\right)^{2},
\end{aligned}
$$

where $\mathbf{p}$ and $\mathbf{q}$ are parameters of the $M_{A}^{(Y)}$ and $M_{Z}^{(Y)}$ models respectively, $(Y)$ indicates the version of the model used for fitting, $N$ is the number of pointing measurements, $\sigma_{Z_{i}}^{2}$ quantifies the $i$ 'th measurement noise level and $w_{i} \sim S_{i}$ is proportional to the radio source flux density $\left(S_{i}\right)$. In practice, we assume $w_{i} / \sigma_{i}=$ $\log \left(\mathrm{SNR}_{i}\right)$, where $\mathrm{SNR}$ is the signal to noise ratio estimated from each pointing measurement.

The choice of the weighting function is to give stronger $\chi^{2}$ contributions from more reliable measurements while accounting for the steep spectrum of the distribution of the intrinsic flux densities in the observed radio source population. This choice may impact the reconstructed confidence intervals, but as long as the numerical precision is not a concern (and for the assumed Markov chain convergence criteria) the choice should not affect the best fit solutions. In this work, we are only concerned with finding the best fitting model through $\chi^{2}$ minimization, and we do not reconstruct the parameter posterior distributions.

In the fast_track version of the control system, the coordinates $\left(A_{E}, Z_{E}\right)$ and the values $C_{A}\left(A_{E}, Z_{E}\right)$ and $C_{Z}\left(A_{E}, Z_{E}\right)$ for any given measurement are not directly available from observations. We therefore use the following approximation:

$$
\begin{aligned}
& \epsilon_{A}\left(A_{E}, Z_{E}\right) \approx \Delta A+C_{A}\left(A_{\mathrm{oa}}, Z_{\mathrm{oa}}\right) \\
& \epsilon_{Z}\left(A_{E}, Z_{E}\right) \approx \Delta Z+C_{Z}\left(A_{\mathrm{oa}}, Z_{\mathrm{oa}}\right) .
\end{aligned}
$$

In order to derive the $\chi^{2}$ values for any given set of parameter values, a similar approximation is used to calculate the new pointing model corrections $\left(M_{A}\right.$ and $\left.M_{Z}\right)$ at $\left(A_{E}, Z_{E}\right)$. While the transformation from $\left(A_{T}, Z_{T}\right)$ to $\left(A_{\mathrm{oa}}, Z_{\mathrm{oa}}\right)$ coordinates is possible by reconstructing the weather dependent refraction history $(1 \mathrm{a}, \mathrm{b})$, in the current work we perform this transformation using the mean refraction model $(13 \mathrm{a}, \mathrm{b})$ which is sufficient given the quality of the present data, and the fact that the data cover $Z_{T}<80^{\circ}$ where $R<0.15^{\circ}$. The COR corrections in this range are even smaller.

When calculating $\chi^{2}$ values we make at least two implicit approximations that should be addressed. One results from calculating the model position correction 
values at wrong directions: $\left(A_{\mathrm{oa}}, Z_{\mathrm{oa}}\right)$ rather than $\left(A_{E}, Z_{E}\right)$, and the other stems from associating the measured corrections $\left(\Delta_{A}, \Delta_{Z}\right)$, to wrong directions: $\left(A_{\mathrm{oa}}, Z_{\mathrm{oa}}\right)$, rather than $\left(A_{E}, Z_{E}\right)$. This is expressed in $(8 \mathrm{a}, \mathrm{b})$. The approximation is justified by the fact that the fastest recorded changes of the position corrections are about 0.003 $\mathrm{deg} / \mathrm{deg}$, which when converted to the angular scales of the differences between $\left(A_{E}, Z_{E}\right)$ and $\left(A_{T}, Z_{T}\right)$ coordinates (even at those lowest elevations), the approximation gives errors of the order $0.0003^{\circ}$ at the most. In practice, the errors are much smaller because "Model 4c" does not model the fastest variations of the corrections associated with rail irregularities, and the rate of correction changes in this model is actually much smaller than the measured value. Therefore, for the current data the error due to this approximation is not important.

Except for the rail irregularity, the effects associated with pointing corrections described in Section 2 can be accurately modeled by slightly modified formulas derived in [4]:

$$
\begin{aligned}
& M_{A}^{(4 e)}\left(\mathbf{p}, A_{\mathrm{oa}}, Z_{\mathrm{oa}}\right)=A_{0}+\left(\xi_{A} \sin \left(A_{\mathrm{oa}}\right)-\zeta_{A} \cos \left(A_{\mathrm{oa}}\right)+\sigma\right) \cot \left(Z_{\mathrm{oa}}\right)+\frac{\beta}{\sin \left(Z_{\mathrm{oa}}\right)}+p_{1} \sin \left(2 A_{\mathrm{oa}}\right) \\
& +p_{2} \cos \left(2 A_{\text {oa }}\right)+p_{3} \sin \left(3 A_{\text {oa }}\right) \cos \left(Z_{\text {oa }}\right)+p_{4} \cos \left(A_{\text {oa }} / 4\right) \sin \left(Z_{\text {oa }}\right) \\
& M_{Z}^{(4 e)}\left(\mathbf{q}, A_{\text {oa }}, Z_{\text {oa }}\right)=Z_{0}+\xi_{Z} \cos \left(A_{\text {oa }}\right)+\zeta_{Z} \sin \left(A_{\text {oa }}\right)+\gamma \sin \left(Z_{\text {oa }}\right) \\
& +q_{1} \cos Z_{\mathrm{oa}}+q_{2} \sin \left(2 A_{\mathrm{oa}}\right)+q_{3} \cos \left(2 A_{\mathrm{oa}}\right)
\end{aligned}
$$

where $\mathbf{p}=\left\{A_{0}, \xi_{A}, \zeta_{A}, \sigma, \beta, p_{1}, p_{2}, p_{3}, p_{4}\right\}$ and $\mathbf{q}=\left\{Z_{0}, \xi_{Z}, \zeta_{Z}, \gamma, q_{1}, q_{2}, q_{3}\right\}$ are the model parameters. This 16-parameter model is a small angle limit of the exact "Model 4c", but extended by an additional ad-hoc parameter $q_{3}$, which proves to be useful in mitigating large-scale trends in zenith distance residuals. The model has a simpler formulation than the full "Model $4 \mathrm{c}$ " $\left(M^{(4 c)}\right)$, but retains a very high compatibility with it (Borkowski, 2016, private communication). We will hereafter refer to this model as "Model $4 \mathrm{e}$ " or $M^{(4 e)}$. In the original version of model $M^{(4 c)}$, the parameter $q_{3}=0$ and the equations are coupled since $\xi_{A}=\xi_{Z}=\xi$ and $\zeta_{A}=$ $\zeta_{Z}=\zeta$, which is physically justified.

The $A_{0}$ and $Z_{0}$ are simple constant offsets, $\gamma$ calibrates the gravitational sag of the secondary mirror and its supports, $\xi$ and $\zeta$ parameters define tilt angles of the telescope azimuth axis towards local meridian and towards the West (the so-called tilt-over and tilt-out components), $\sigma$ defines angle between the elevation axis and the plane of local horizon, and $\beta$ is the angle between plane perpendicular to elevation axis and the optical axis. Seven ad-hoc terms $\left(p_{1}, p_{2}, p_{3}, p_{4}\right.$, and $\left.q_{1}, q_{2}, q_{3}\right)$ are inserted empirically to accommodate for corrections that cannot be modeled by the aforementioned construction imperfections, but may be related with e.g. eccentricity of gearwheels etc.

When the equations are coupled $\left(\xi_{A}=\xi_{Z}=\xi\right.$ and $\left.\zeta_{A}=\zeta_{Z}=\zeta\right)$ the two models $\left(M_{A}\right.$ and $\left.M_{Z}\right)$ can be fitted jointly by minimizing:

$$
\chi^{2}=\chi_{A}^{2}+\chi_{Z}^{2}
$$

however, we find that the resulting best-fit model yields residuals a factor of $\sim 1.7$ times larger in zenith distance and $\sim 1.1$ times larger in azimuth, than in the case when the two parameters are allowed to differ between the models for each of the 
two coordinates. We will therefore use the model with 16 parameters as defined in (9a,b), even though it is not as well physically motivated.

\section{Observations}

Between April and October 2016 we carried out a pointing campaign using OCRA$\mathrm{p}$ [6], a 30-GHz dual beam, beam-switched receiver with half power beamwidth $\theta_{\text {OCRA }} \approx 1.2^{\prime}$ and with the reference beam offset

$$
\left(B_{A}, B_{Z}\right)_{\text {OCRAp }}=\left(0.432^{\circ},-0.0720^{\circ}\right) .
$$

During the observations the pointing model "Model 4c" (Section 3) was used along with the fast_track version of the control system. The observations were carried out using the "ocraToolkit" software package, designed for OCRA-SZ observational project [18-20].

For any given radio source, we perform a cross-scan (Fig. 1) as previously discussed in [23] and [9], and we extract the position corrections $\left(\Delta_{A}, \Delta_{Z}\right)$ (Fig. 2) by fitting gaussian and double gaussian functions to the data points after having removed drifts arising due to atmospheric effects [21]. The averaged cross-scan data have 1-s time resolution.

We observed a sample of 26 distinct radio sources (Fig. 3). The sample is composed mostly of active galactic nuclei (AGNs) including: intermediate to high redshift $(0.2<z<2.5)$ quasars $(14)$, and low to intermediate redshift $(0.017<z<0.72)$ Seyfert galaxies (6), BL Lac objects (4), a radio galaxy (1) and the NGC 7027 planetary nebula, all of which should appear a point-like with OCRA-p beam. However, the majority of observations (92\%) were performed using a small subgroup of brightest sources (9). By analyzing the the quality of the fits of the model beam to the data, as well as the repeatability of the corrections within ranges where they vary slowly, we estimate that for the data with high SNR the uncertainty of position correction determination is small $O\left(10^{-4}\right) \mathrm{deg}$, but possibly up to $\approx 0.004 \mathrm{deg}$ for poor weather or low SNR.

For the pointing campaign the control system has been modified to account for a number of astrometric effects which are relevant at the milli-degree pointing accuracy. The modifications include: (i) introducing UT1 time scale ${ }^{1}$ which gives an effect of $<0.6 \mathrm{~s}$ in hour angle, (ii) adapting the nutation model as implemented in the NOVAS library [16] ${ }^{2}$ which affects pointing at the level $\lesssim 5 \mathrm{mdeg}$, (iii) introducing weather dependent radio refraction as implemented in SLA library [37] with weather data based on real-time readouts from the local meteorological station (effect of $\lesssim 50$ mdeg for $Z<80$ and relevant meteorological conditions), (iv) enabling annual

\footnotetext{
${ }^{1}$ All time-related operations in the control system are based on UT1 time scale. The UT1 time is updated regularly using the data available online at http://maia.usno.navy.mil/ser7/mark3.out.

${ }^{2}$ http://aa.usno.navy.mil/software/novas/novas_info.php. While the previous implementation of the nutation was consistent with NOVAS library at the level $<10^{-5} \mathrm{deg}$, it was not used due to bugs introduced during porting the code from FORTRAN to C.
} 


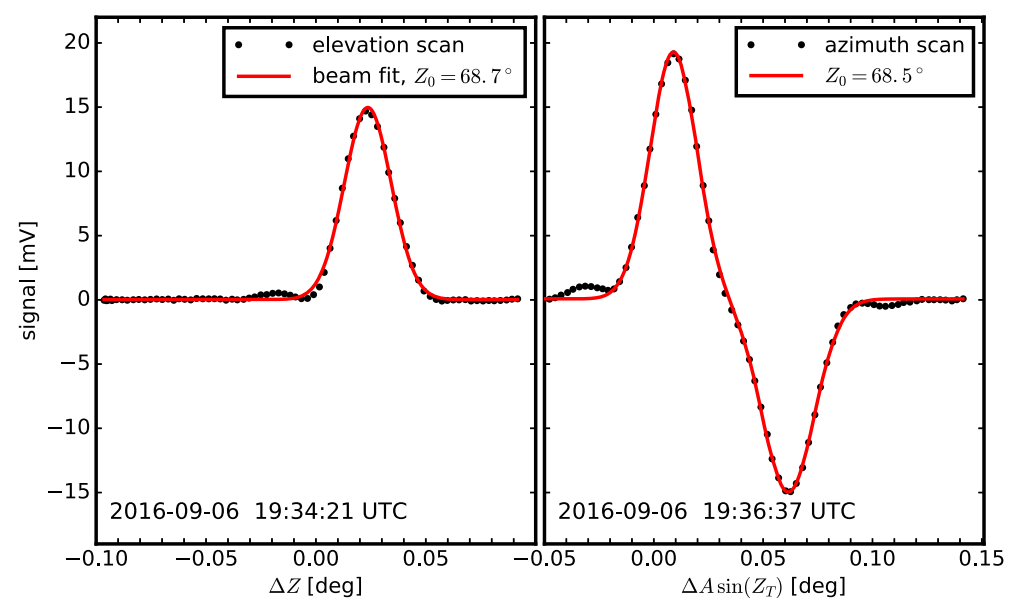

Fig. 1 A high signal to noise (SNR $\approx 510$ ) cross-scan observation of 3C 84 with a linear drift removed. First, elevation scan is performed (left) and it is followed by azimuth scan (right), accounting for the position correction from the elevation scan. Side lobes of up to $5 \%$ are asymmetric due to receiver beam offset (11)

aberration in coordinate transformations ${ }^{3}$ (effect of $\lesssim 6 \mathrm{mdeg}$ ), and (v) correcting the telescope geodetic coordinates to match those obtained from a geodetic VLBI experiment [7], which gives an effect of $\approx 0.72 \mathrm{~s}$ in hour angle.

We have also improved the observing strategy that exploits, the possibility of controlling the velocity of the telescope drives in azimuth and elevation in order to scan a source along one coordinate while tracking, rather than imposing a series of fixed offsets that are reached along an unpredictable trajectory. This also allows us to directly control the cross-scan speed. It was estimated that for any given cross-scan these improvements provide about $10 \%$ larger receiver response due to the radio source passing closer to the beam center as the telescope sweeps the sky during the azimuth scan.

Another improvement over the previous pointing observations, comes from a higher time resolution (roughly $8 \mathrm{~Hz}$ ) of the telescope position readouts obtained from the RTLinux based control system (a factor of $\sim 8$ improvement) and also from the way the position data are matched to the signal stream - i.e. by using an interpolation rather than the nearest neighbor approach.

\section{Data processing}

The pointing data are a compilation of cross-scan observations of selected radio sources, observed over ranges of hour angles with a typical time lapse between the adjacent observations of $\sim 5$ minutes. Depending on the type of observational program, the time elapsed between subsequent cross-scan observations of

\footnotetext{
${ }^{3}$ The diurnal aberration and polar motion are currently neglected.
} 


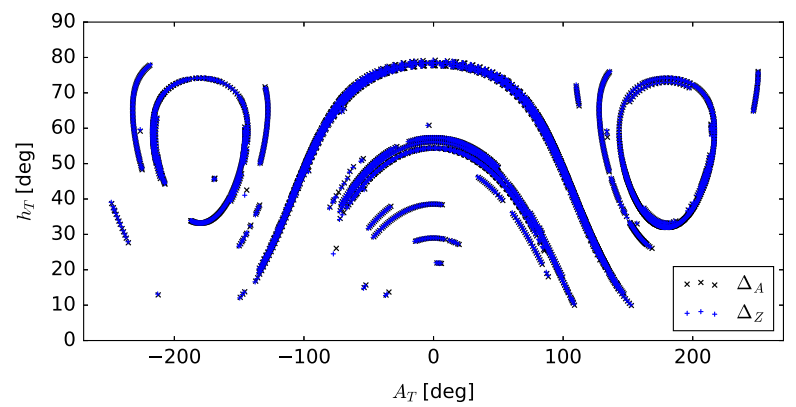

Fig. 2 Pointing corrections in azimuth (top) and zenith distance (bottom) obtained from pointing observations as a function of azimuth and zenith distance

the same source can increase to several minutes. Each scan record contains $\left\{A_{T}, Z_{T}, \alpha_{T}, \delta_{T}, t, \Delta, \mathrm{SNR}\right\}$ (Table 1), where $t$ is the UT1 time of the source maximal response, $\Delta$ is the derived position offset in azimuth or zenith distance and SNR is a signal-to-noise estimator that we use to weight contributions to the $\chi^{2}$ (7a,b). Each cross-scan observation contains two such records: one for the azimuth scan in which case $\Delta \equiv \Delta_{A}$ and one for the elevation scan in which case $\Delta \equiv \Delta_{Z}$.

The cross-scan data are pre-processed before the analysis in several stages. On 14 June 2016 a number of important astrometric corrections have been implemented into the control system (Section 4), therefore in stage-one we select only the data gathered after that date.

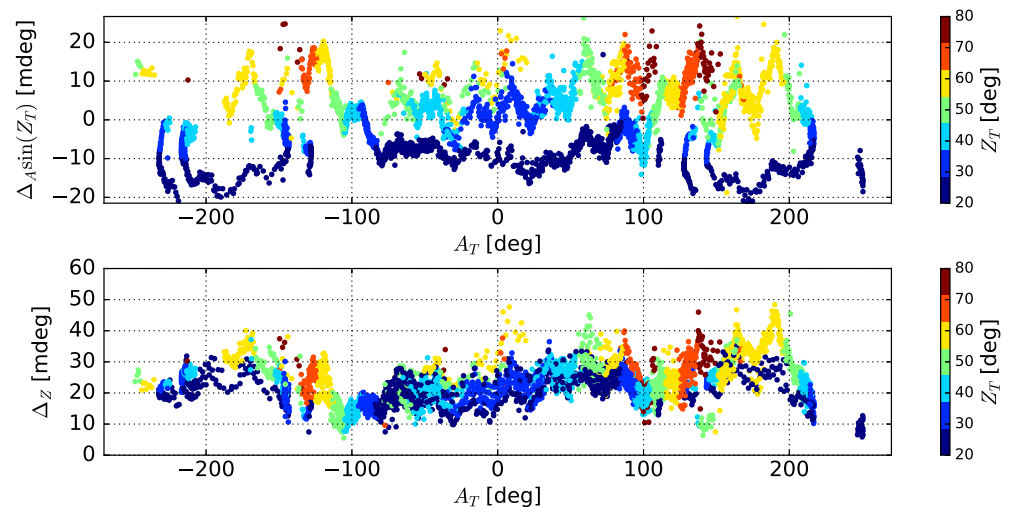

Fig. 3 Distribution of stage-two pointing measurements in azimuth-elevation plane (see Table 1). In most cases the direction of azimuth correction of a given cross-scan is close to the direction of zenith distance correction of the same cross-scan. In some records, this is not the case due to e.g. failure in finding the best-fit position correction in the first iteration. In such cases, the scan is repeated along that coordinate until a satisfactory fitting is achieved a few minutes later 
In stage-two we further screen this data to remove obvious outliers (Fig. 2). This is done by requiring

$$
\begin{aligned}
\left|\Delta_{A}\right| & <0.03^{\circ} \\
0.005^{\circ}<\Delta_{Z} & <0.05^{\circ}
\end{aligned}
$$

This condition leaves 4076 pointing measurements that fully cover the entire range of azimuths and the observationally useful range of zenith distances (Fig. 3). Clearly, for any given $A_{T}$ the pointing corrections depend on $Z_{T}$ as well. However, they do so consistently: i.e. for a given $A_{T}$, negative $\Delta_{A}$ corrections reside typically at low zenith distances (and vice-versa), but not at low and high zenith distances simultaneously, which would hint on data inconsistency or time-dependent effects. The same seems to be true for $\Delta_{Z}$ corrections. A statistic of pointing precision of the fast_track version of the control system is shown in Fig. 4.

In the stage-three of data pre-processing coordinates of each pointing measurement are transformed from $\left(A_{T}, Z_{T}\right)$ to $\left(A_{\text {oa }}, Z_{\text {oa }}\right)$ using OCRA-p receiver beam offset (11) and the mean optical atmospheric refraction according to:

$$
\begin{aligned}
& A_{\mathrm{oa} i}=A_{T i}+B_{A}^{\mathrm{OCRAp}} / \sin \left(Z_{\mathrm{oa} i}\right) \\
& Z_{\mathrm{oa} i}=Z_{T i}+B_{Z}^{\mathrm{OCRAp}}-R\left(Z_{T i}\right) .
\end{aligned}
$$

Using the approximation given in $(8 \mathrm{a}, \mathrm{b})$ we calculate $\epsilon_{A_{i}}\left(A_{\mathrm{oa}}, Z_{\mathrm{oa}}\right)$ and $\epsilon_{Z_{i}}\left(A_{\mathrm{oa}}, Z_{\mathrm{oa}}\right)$. In (13b) we use formula for optical refraction even though radio and weather-dependent refraction model was used during the observations. This shortcoming has little effect on reconstructing $Z_{\mathrm{oa}}$ directions as explained in Section 3.

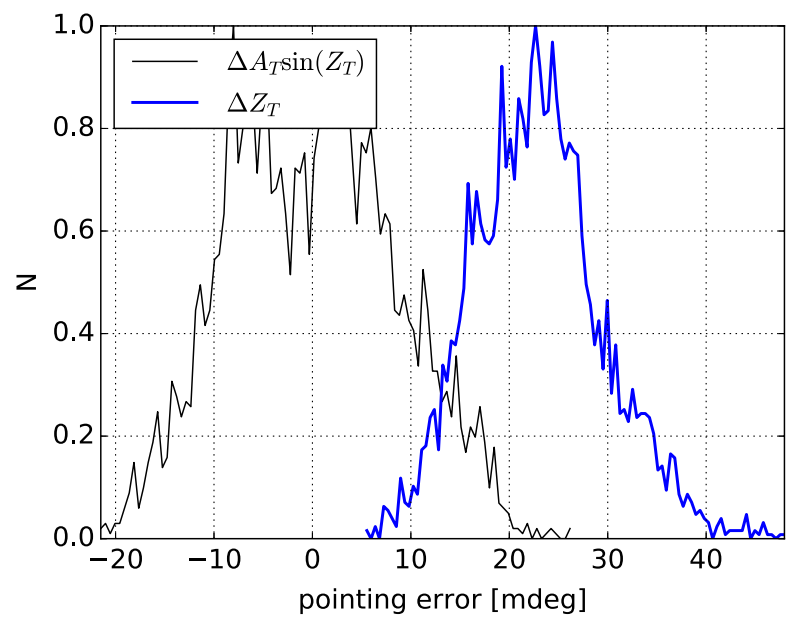

Fig. 4 Distribution of pointing errors from Fig. 2 (Section 5). The root mean square (RMS) values of the pointing corrections are given in Table 2 


\section{Finding the best-fit model}

We fit the 16-parameter model as defined in $(9 a, b)$ to the pointing data discussed in Section 5 using Monte-Carlo Markov-Chain (MCMC) approach, combined with simulated annealing (SA) algorithm. A typical MCMC chain with a moderate cooling rate takes about 20000 to 40000 steps before a converged solution is found. We assume a flat initial prior distribution for each parameter.

The residuals between the pointing data and the best-fit "Model 4e":

$$
\begin{aligned}
& \Delta_{A}^{(4 e)}=\epsilon_{A}-M_{A}^{(4 e)}\left(\mathbf{p}, A_{\mathrm{oa}}, Z_{\mathrm{oa}}\right) \\
& \Delta_{Z}^{(4 e)}=\epsilon_{Z}-M_{Z}^{(4 e)}\left(\mathbf{q}, A_{\mathrm{oa}}, Z_{\mathrm{oa}}\right)
\end{aligned}
$$

are shown in Fig. 5.

The figure clearly shows the possibility of improving the telescope pointing precision. The zoom-in panels also show, that what looks like a noise (left plots in Fig. 5) actually has a fine structure that is resolved with the current quality of the data (e.g. Fig. 5 top-right). The statistical error of a pointing measurement, $O\left(10^{-4}\right)$ degree, is much smaller than the systematic errors still present in $\Delta^{(4 e)}$ residuals and clearly, model $M_{A}^{(4 e)}$ is unable to fit them. This indicates that the pointing model (9a,b) can be further improved. The residuals also confirm that the rate of variation of the azimuth corrections is $<3 \mathrm{mdeg} / \mathrm{deg}$.

It is clear that for any given range of azimuths and/or elevations the dispersion of the zenith distance pointing corrections is larger, than it is in the case of azimuth corrections (Fig. 5). This may indicate problems of repeatability of the measurements (e.g. resulting from the stability of the suspension of the secondary mirror). For certain ranges of azimuths, the azimuth or elevation position corrections may vary by as much as $\sim 0.015^{\circ}$ for two distinct elevations or distinct measurement dates. This also hints that some effects yet unaccounted for may play some role.
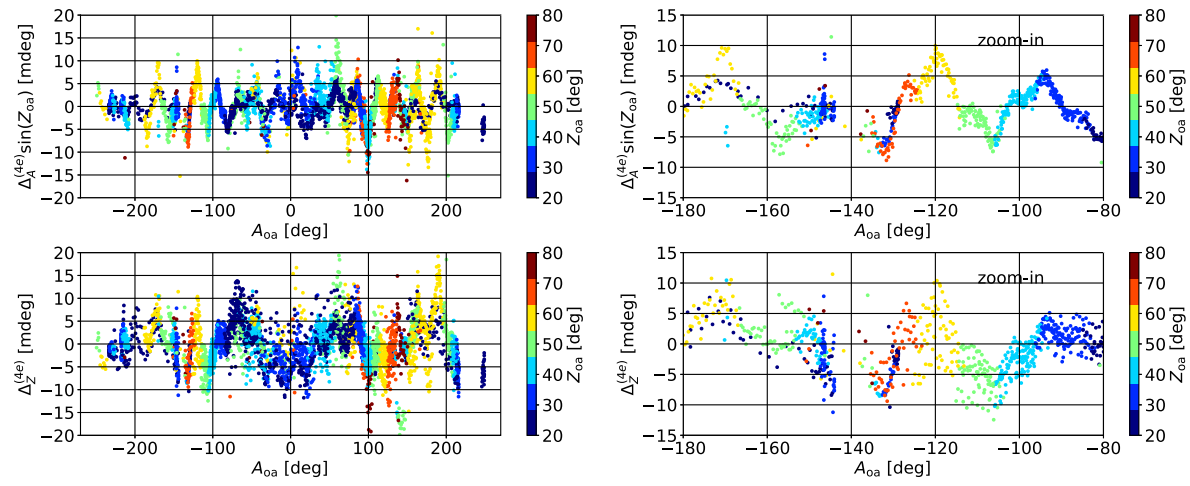

Fig. 5 Residuals between the pointing data and the best fit model "Model 4e" (left) and a zoom-in region (right). Notice, that for certain azimuth ranges the position corrections differ depending on zenith distance of the source used for measurement, whereas in other ranges there is a good overlap regardless of the source elevation 


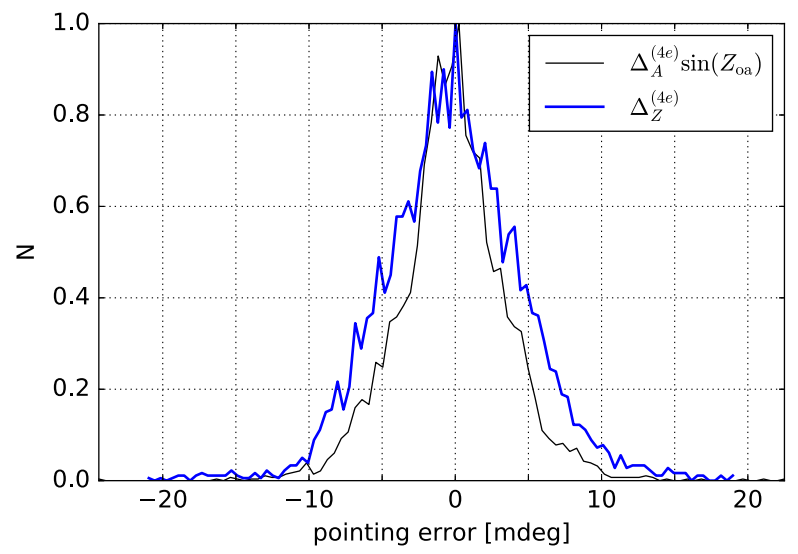

Fig. 6 Distribution of "Model 4e" residuals from Fig. 5. The RMS values of the residuals are given in Table 2

The best fit parameter values in scientific notation in degrees yield:

$$
\begin{aligned}
\mathbf{p}= & \{3.414471 \mathrm{e}-03,-9.148816 \mathrm{e}-04, \\
& -2.298499 \mathrm{e}-03,1.886013 \mathrm{e}-02, \\
& -4.381365 \mathrm{e}-02,-1.035695 \mathrm{e}-02, \\
& 8.263103 \mathrm{e}-03,-7.497834 \mathrm{e}-03, \\
& 5.051955 \mathrm{e}-03\}
\end{aligned}
$$

Table 2 Summary of measured and projected 32-m Torun radio telescope pointing precision

\begin{tabular}{llllll}
\hline Control system & Pointing & RMS $(A)$ & RMS $(Z)$ & \multicolumn{2}{l}{ Improvement $^{\mathrm{c}}$} \\
\cline { 5 - 6 } version & Model & {$[\mathrm{mdeg}]$} & {$[\mathrm{mdeg}]$} & $A$ & $Z$ \\
\hline fast_track & Model 4c & 8.5 & 24.1 & 1.0 & 1.0 \\
COCONUT $^{\mathrm{a}}$ & Model 4e & 4.1 & 7.9 & 2.1 & 3.1 \\
COCONUT $^{\mathrm{b}}$ & Model 4e & 3.7 & 4.8 & 2.3 & 5.0 \\
COCONUT $^{\mathrm{a}}$ & Model 5 & 2.2 & 3.4 & 3.9 & 7.1 \\
COCONUT $^{\mathrm{d}}$ & Model 5 & $2.7(2.1)$ & $3.9(3.2)$ & 3.1 & 6.2 \\
\hline
\end{tabular}

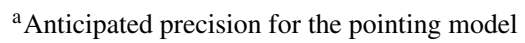

${ }^{\mathrm{b}}$ The best fit assuming $\xi_{A}=\xi_{Z}=\xi$ and $\zeta_{A}=\zeta_{Z}=\zeta$ in $(9 \mathrm{a}, \mathrm{b})$

${ }^{c}$ RMS improvement with respect to the fast_track version of the control system

${ }^{\mathrm{d}}$ Precision measured using an additional pointing data taken after the pointing model was implemented into the control system. Standard deviations are also given in parentheses for comparison, in order to indicate yet unidentified, long-term systematic effects that are still not accounted for in the model (see Fig. 10 and Section 9 for a discussion) 


$$
\begin{aligned}
\mathbf{q}= & \{8.268194 \mathrm{e}-02,4.271137 \mathrm{e}-05, \\
& 2.704925 \mathrm{e}-04,-8.758806 \mathrm{e}-04, \\
& -3.489975 \mathrm{e}-02,-4.142412 \mathrm{e}-03, \\
& 3.697197 \mathrm{e}-03\}
\end{aligned}
$$

The anticipated improvement due to introducing model $M^{(4 e)}$ is shown in Fig. 6 and in Table 2.

\section{Modeling rail surface irregularities}

The fluctuations of $\Delta^{(4 e)}$ are largely independent from the zenith distance coordinate (Fig. 5), thus introducing further improvements simpler, as compared to the case when the corrections or residuals depend on azimuth and elevation at the same time.

In order to further improve "Model 4e", we (i) wrap $\left(\Delta_{A}^{(4 e)} \sin \left(Z_{\mathrm{oa}}\right), \Delta_{Z}^{(4 e)}\right)$ residuals into $A=\left[-180^{\circ}, 180^{\circ}\right.$ ) range, (ii) densely resample the corrections on a uniform grid using a linear interpolation, and (iii) decompose them into Fourier series. The Fourier expansion yields:

$$
\delta_{X}\left(A_{\mathrm{oa}}\right)=\delta_{X 0}+\sum_{i=1}^{N_{F}} a_{X, i} \sin \left(\frac{2 \pi}{T_{X, i}}\left(180-A_{\mathrm{oa}}\right)+\phi_{X, i}\right)
$$

where $X=A$ for azimuth residuals and $X=Z$ for zenith distance residuals, $\delta_{X 0}=$ $\left\langle\Delta_{X_{i}} s_{X_{i}}\right\rangle_{i} \approx 0$ where $s_{X_{i}}=\sin Z_{\mathrm{oa}, i}$ for $X=A$ and $s_{X_{i}}=1$ for $X=Z$ and $\Delta_{X_{i}}$ is the $i$ 'th residual calculated using $(14 \mathrm{a}, \mathrm{b})$. We find that $N_{F}=50$ gives a reasonable fit to the model $M^{(4 e)}$ residuals (Fig. 7) and allows us to reconstruct the structures that are well seen in Fig. 5 (zoom-in panels). The values of the coefficients $\left\{T_{i}, a_{i}, \phi_{i}\right\}$ are provided as an attachment to the on-line version of this article.
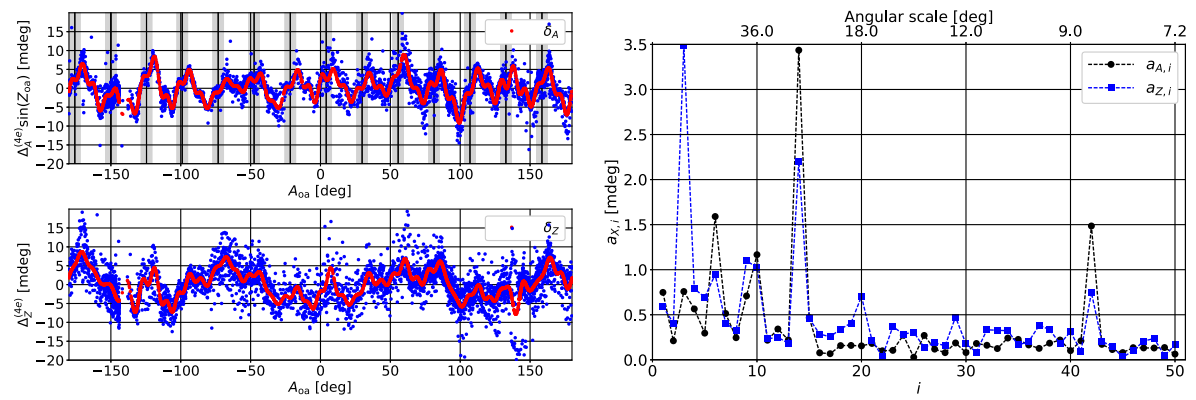

Fig. 7 (Left) Residuals between the pointing data and the best fit model "Model 4e" wrapped into $A=$ $\left[-180^{\circ}, 180^{\circ}\right)$ range (blue) and a Fourier expansion fit based on (16) (red). Vertical lines in the top panel mark the azimuths of the rim welding points, and the shaded bands $\left(\approx 7.6^{\circ}\right.$ wide $)$ correspond to the angular separation of the wheels in each of the four trolleys supporting the telescope. (Right) Fourier expansion coefficients $a_{X, i}$ as defined in (16). High amplitude modes clearly reach into small angular scales, and many of the weaker modes have their phases strongly correlated, which builds up the integrated corrections 

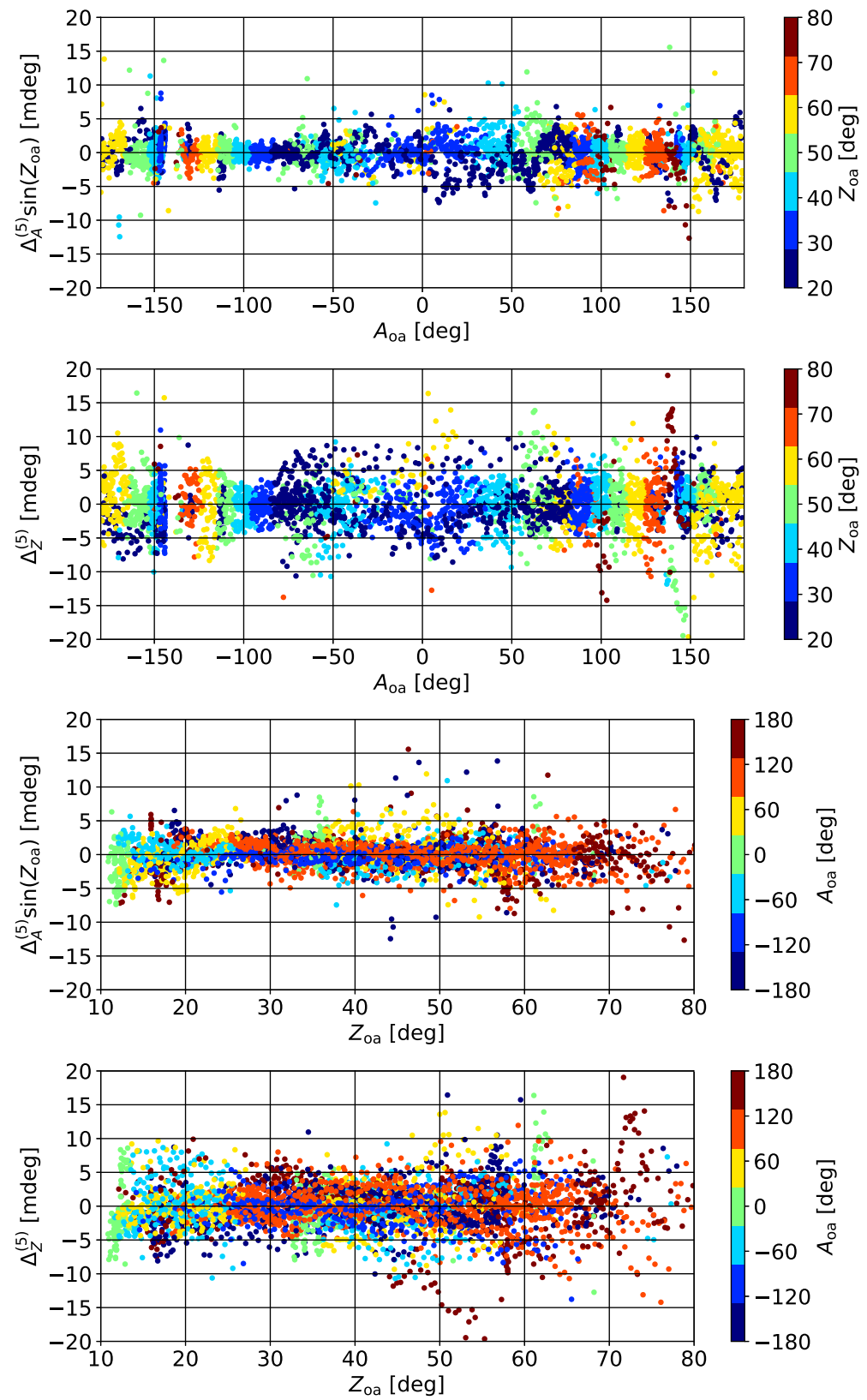

Fig. 8 Residuals between the pointing data and the best fit model "Model 5" $\left(M^{(5)}\right)$ plotted versus azimuth (upper panels) and zenith distance (lower panels). The model effectively removes all systematic effects associated with rail surface irregularities (see Fig. 7) 


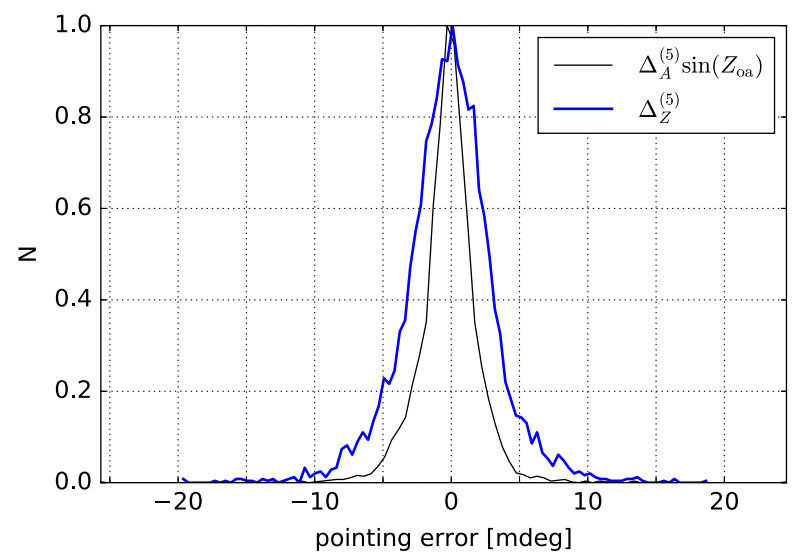

Fig. 9 Distribution of "Model 5" residuals from Fig. 8. The RMS values of the residuals are given in Table 2

We introduce "Model 5" $\left(M^{(5)}\right)$ as:

$$
\begin{aligned}
& M_{A}^{(5)}\left(\mathbf{p}, A_{\mathrm{oa}}, Z_{\mathrm{oa}}\right)=M_{A}^{(4 e)}\left(\mathbf{p}, A_{\mathrm{oa}}, Z_{\mathrm{oa}}\right)+\delta_{A}\left(A_{\mathrm{oa}}\right) \\
& M_{Z}^{(5)}\left(\mathbf{q}, A_{\mathrm{oa}}, Z_{\mathrm{oa}}\right)=M_{Z}^{(4 e)}\left(\mathbf{q}, A_{\mathrm{oa}}, Z_{\mathrm{oa}}\right)+\delta_{Z}\left(A_{\mathrm{oa}}\right)
\end{aligned}
$$

and we reprocess the corrections to obtain new residuals (Fig. 8) and their distribution (Fig. 9). In order to implement this model into a new version of the control system that we call COCONUT, we use the approximation given in $(8 \mathrm{a}, \mathrm{b})$ since the control system calculates the corrections as a function of the telescope encoder coordinates $\left(A_{E}, Z_{E}\right)$.

The large-scale fluctuations are clearly visible in "Model $4 \mathrm{e}$ " residuals and they can be associated with the irregularities of the rail, that is welded out of 14 pieces, which implies a period of $\sim 25.7^{\circ}$. This period matches well the quasi-periodic structures evident in Fig. 5 (top-right) and coincides with the number and the locations

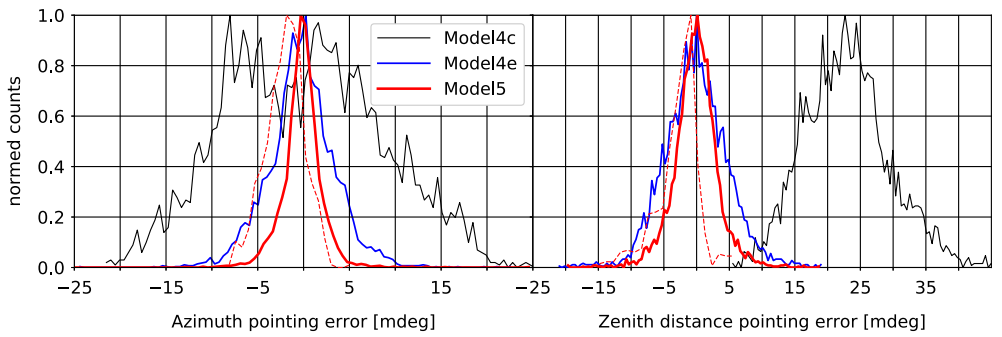

Fig. 10 Comparison of the measured and anticipated pointing errors distributions, assembled from Figs. 4, 6 and 9 , and resulting from different pointing models. The azimuth pointing errors are scaled by $\sin \left(Z_{\mathrm{oa}}\right)$. The RMS values for different pointing models are given in Table 2. The dashed line is a distribution of pointing corrections recorded after "Model 5" has been implemented in the telescope pointing system, and it represents an additional validation of the model. A small, $<1.6 / 1.0 \mathrm{mdeg}$ systematic offset is present in azimuth/elevation, witch can be associated with time-dependent effects that are being analyzed and briefly discussed in Section 9 
of the welding points along the rail (Fig. 7). These saw-tooth-like irregularities were previously detected in the pointing data analyzed by [5], but the quality of that data was substantially worse, and these effects have never been accounted for in any pointing corrections model. A higher frequency modes of up to $\sim 7^{\circ}$ deg are now also evident, and the current data allows us to model them as well. The highest frequency modes that we model also approximately correspond to the angular separation of the wheels of the telescope trolleys.

In Fig. 10 we show a comparison between the distributions of the measured position corrections in the fast_track version of the control system and the anticipated improvements due to using the new pointing models ("Model 4e" and "Model 5"). The improvements are also shown by the RMS values in Table 2.

\section{Measurements of rail and wheels irregularity}

If the telescope wheels are not exactly round, but rather oval to the first approximation, then the fast running pointing corrections may only partially be associated with the rail irregularities as the wheels of $1.4 \mathrm{~m}$ in diameter cover azimuth range of about $21^{\circ}$ per single rotation. ${ }^{4}$ Furthermore, the time stability of the rail model (Fig. $7,(16)$ ) would be uncertain if e.g. wheels could slip over the rail, or roll along non-repeatable paths. In fact, we observed that matching a pair of points, one on the rim of a given wheel and another one on the rail, and both fixed at the same azimuth, is not stable in time.

Therefore, we measure the wheel and rail irregularities using an analog distance indicator, which offers a relative distance measurement accuracy of $0.01 \mathrm{~mm}$. In each case the distance indicator is attached to the trolley chassis (Fig. 11). For the case of wheels, the indicator probe is set against the wheel surface and points at its center. As the telescope moves a video camera records the probe indications and the recording is repeated for each wheel. For the case of the rail, the probe is fixed vertically against the rail surface and the recording is done for two dish orientations: $Z=0^{\circ}$ and $Z=60^{\circ}$. We measure the rail deformations with the probe installed at two different trolleys: front and back, at two different distances from the wheel: $\sim 60 \mathrm{~cm}$ and $\sim 140$ cm respectively.

The azimuthal extent of rail deformations is unknown, and in our measurement setting (Fig. 11) the indicator readings depend on the distance of the probe from the wheel. For the case when the probe is fixed nearby the wheel, we anticipate that the measured value of the vertical rail deformation $(\Delta h)$ is biased low, since in this case the probe will capture only a fraction of the full indent. In the extreme case, if the probe was set to measure the distance variations (from the chassis to the rail) right under the wheel, no variation would be detected (assuming that wheels are round). The distance of $60 \mathrm{~cm}$ from the wheel is about a factor of 2 smaller than the distance that corresponds to the azimuth range $\left(\sim 7^{\circ}\right)$ over which we observe

\footnotetext{
${ }^{4}$ The highest frequency Fourier modes that model the rapidly changing position corrections have period of about $7^{\circ}$ (Section 7 ).
} 

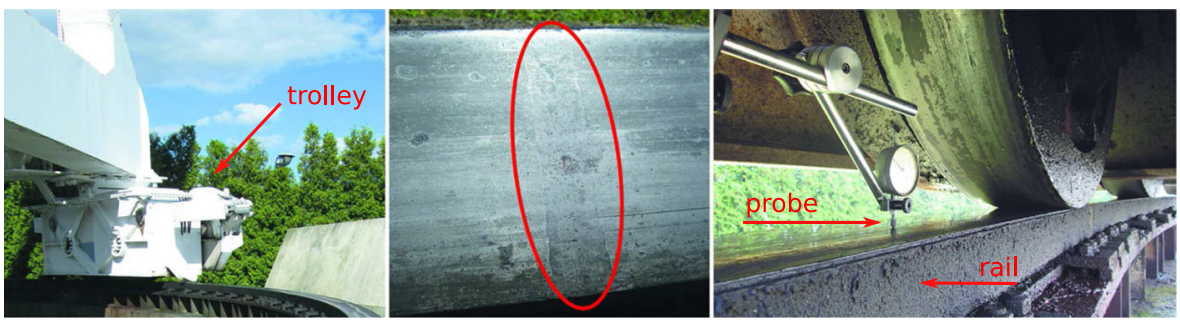

Fig. 11 Rail surface irregularity measurement. The response of the rail to the pressure induced by twowheeled trolleys supporting the telescope structure (left) is measured for all azimuths using readings from an analog distance indicator attached to the trolley chassis (right). The largest variations are observed in the vicinity of rail welding points (center)

significant variations in the measured position corrections, which we associate with the rail irregularity (Fig. 7). Therefore, we also measure the track height variations with the probe fixed about $1.4 \mathrm{~m}$ away from the wheel. At this distance we expect to capture most of the rail indents.

An analysis of the relative variations of the wheels radii, shows that the maximal deviation from the circular shape is $<0.05 \mathrm{~mm}$. This is negligible when compared to the amplitude of the rail indents around the welding points: $<2.7 / 4.4 \mathrm{~mm}$ with the probe fixed at the distance of about $60 / 140 \mathrm{~cm}$ from the wheel in one of the front/back trolleys. We find that the dependence of these values on the dish elevation is weak (a difference of $<0.2 \mathrm{~mm}$ between $Z=0^{\circ}$ and $Z=60^{\circ}$ ).

Assuming that a rail indent of $\approx 4.4 \mathrm{~mm}$ can generate a pointing offset of up to $\sim 20$ mdeg (Fig. 7), it should be expected that the effects due to non-round wheels will limit the pointing accuracy to $<0.25 \mathrm{mdeg}$. This is below the pointing precision we aim at, and therefore the effects due to non-round wheels can be neglected at the present.

\subsection{Impact on pointing corrections}

It is instructive to consider a toy model of rail surface instability in order to estimate the expected amplitude of the associated pointing corrections.

The radius of the rail is $R=12 \mathrm{~m}$, and the four trolleys supporting the telescope structure are located at corners of a square frame (Fig. 11). Assuming that the telescope structure is rigid and that one of the trolleys is rolling down a local rail indent of the amplitude $\Delta h$, the opposite trolley will be lifted (or will be less loaded) and the vertical axis of the telescope will be rotated about the axis defined by the two remaining trolleys. The rotation angle will be:

$$
\gamma=\arcsin \left(\frac{\Delta h}{R}\right) \approx 4.8\left(\frac{\Delta h}{1 \mathrm{~mm}}\right)[\mathrm{mdeg}]
$$

Assuming that the largest amplitude of the rail indents (registered over the full azimuth rotation) is $\Delta h \approx 4.4 \mathrm{~mm}$, the expected $\gamma$ is about $21 \mathrm{mdeg}$. It is 
straightforward to calculate (Appendix) that, in the limit of small angles, the associated pointing corrections are:

$$
\begin{aligned}
\Delta A \sin (Z) & =\gamma \cos (Z) / \sqrt{2} \\
\Delta Z & =\gamma / \sqrt{2},
\end{aligned}
$$

which gives the expected amplitude of the corrections at the level $<15$ mdeg in both coordinates.

In Fig. 7 the amplitude of the systematic effects, that we associate with rail surface irregularities, ranges from about $16 \mathrm{mdeg}$ to $18 \mathrm{mdeg}$ for elevation and cross-elevation respectively.

Since these values are within a factor of $\sim 1.2$ from the values predicted by the model at the zenith, it is clear that the toy model, given a realistic rail indent amplitude measurement, predicts the pointing corrections that are quite consistent with the observations discussed in the previous sections (Section 7). However, the effects of rail indents are operating in all four trolleys simultaneously and possibly are also associated with some structural deformations, therefore in this work we model the position corrections due to the rail instability relying solely on pointing measurements.

\section{Discussion}

Although "Model 5" predicts an improvement in pointing precision by a factor of a few with respect to the fast_track version of the control system (Table 2), Fig. 8 also hints that the pointing data, when corrected for the best-fit pointing model $\left(M^{(5)}\right)$, may still have some residual zenith distance dependence. For example, in the azimuth range $A=\left[50^{\circ}, 70^{\circ}\right]$ the azimuth residuals seem to be smaller at lower elevations than the residuals of the measurements taken at higher elevations. The same seems to be true for the zenith distance residuals in the azimuth range $A=\left[-180^{\circ},-150^{\circ}\right]$. Whether this is a coincidence is not clear at the present. The azimuth and zenith distance residua (Fig. 8) clearly depend on elevation around $A=140^{\circ}$. New pointing data may help to better understand the remaining issues around those and other directions.

The data presented in this paper are the most accurate pointing observations ever collected with 32-m Torun radio telescope (Fig. 5 right panels). However, the noise level of the azimuth residuals is not uniform across the full range of azimuths (Fig. 8). Generally, the azimuth residuals have smaller dispersion than the zenith distance residuals. It remains to be seen whether the larger dispersion in zenith distance can be associated with imperfections in fixation of the secondary mirror. Taking into account results from independent observations of the position of the secondary mirror, which are performed using an optical camera installed in the secondary focus cabin, this possibility seems plausible. However, by inspecting the residuals in Fig. 8, it is clear that at the current noise level, no obvious nor strong large-scale systematical effects are present, which suggests that the extended pointing model $(17 \mathrm{a}, \mathrm{b})$ accounts for all major construction deficiencies. 
In principle, the dimensionality of the model parameter space could be reduced by utilizing independent measurements of the gravitational sag, that can be obtained with aid of the large-zoom optical camera, installed in the secondary focus cabin, which we use for real-time monitoring of the position of the secondary mirror. However, the gravitational sag can be a function of temperature and it is possible that a general solution may require a more complicated model of the sag. We will investigate this possibility in another study (B.S. Lew, 2018, in preparation).

The pointing models considered in this work do not accommodate for horizontal focus box offsets that depend on elevation, nor for non-vanishing azimuth dependence of the gravitational sag. Results from optical imaging suggest that such effects may be present, but they are not dominant. The anticipated pointing precision of the COCONUT version of the control system $(<3.4 \mathrm{mdeg})$ is still at least a factor of a few below the tracking capability of the telescope. Yet higher accuracy pointing data would be needed to explore these possibilities in greater details and possibly further improve the pointing.

The data analyzed in the this paper (Section 5) do not provide multiple observations of same directions, and the sky coverage is incomplete. With the advent of new pointing data, to be taken with the COCONUT version of the control system (utilizing "Model 5"), it will be possible to verify and investigate the time stability of the corrections, which is of crucial importance for any pointing model that is calibrated against observational data taken over a short time interval. This will also address the important issues of short- and long-term thermal effects on the pointing performance as the amplitude of annual temperature variations at the telescope site typically spans well over $40{ }^{\circ} \mathrm{C}$.

The "Model 5" has been implemented into control system in December 2016 and has been tested since then. The actual pointing measurements taken right after the implementation of the model (but a few months after the acquisition of the data used to calibrate the models presented in this work) confirm the anticipated pointing precision (Table 2). However, when combined with large zoom video camera observations of the secondary mirror position, they also hint at the presence of new long- and short-term thermal or time-dependent systematic effects, which are being analyzed (Fig. 10). For example, if during long integrations the anisotropic illumination by the Sun could induce a temperature difference of $\Delta T=5{ }^{\circ} \mathrm{C}$ between the supporting legs, then the corresponding difference in leg lengths of $\sim 1 \mathrm{~mm}$ should be expected due to thermal expansion. ${ }^{5}$ These effects could translate onto pointing corrections of the amplitude similar to those caused by the rail surface irregularities. Some of the new pointing data also show fast varying, then disappearing pointing abnormalities, but those are typically excluded at the data selection stage. Similar effects have also been previously noted in [2] for a comparable telescope. In such a case, accounting for thermal effects could be important for reaching stable, milli-degree pointing, but this exceeds beyond the scope of the this work.

\footnotetext{
${ }^{5}$ We assume the effective length of the legs of $L \approx 18 \mathrm{~m}$, and the linear thermal expansion coefficient for iron $\alpha=12 \times 10^{-6} \mathrm{~K}^{-1}$.
} 


\section{Conclusions}

We implement a number of astrometric improvements into the control system of the 32-meter radio telescope located near Torun. Using a dedicated software toolkit we acquire pointing data from observations carried out in 2016, and we analyze them in order to improve the pointing precision.

We slightly modify and extend the pointing corrections model used during the data taking, and we refer to it as "Model $4 \mathrm{e}$ ". Using the pointing data and $\chi^{2}$ minimization approach, we find the values of 16 parameters of the model that minimize the pointing correction residuals. The best-fit model can improve the pointing precision to $13^{\prime \prime}\left(17^{\prime \prime}\right)$ in azimuth (elevation) as measured by the RMS of the residual pointing corrections, or by a factor of $2.3(5.0)$ with respect to the pointing precision available at the time of the data taking.

Next, we analyze the pointing corrections processed with "Model $4 \mathrm{e}$ ". We find systematic fluctuations in the residuals with peak-to-peak amplitude of up to $\sim 20$ mdeg, which we identify to be associated with the telescope rail. The rail yields under the weight of 620-ton telescope as four two-wheeled supporting trolleys roll over and around the rail welding points. The pointing effects associated with the rail vertical deformations, which amplitude we measure to be $<4.4 \mathrm{~mm}$, cannot be modeled with "Model 4e". In order to account for these irregularities we introduce an extension to the model that we call "Model 5". The extension allows us to further improve the pointing accuracy down to $8^{\prime \prime}\left(12^{\prime \prime}\right)$ in azimuth (elevation) or by a factor 1.7 (1.4) with respect to the best fit model ("Model 4e") that does not account for the rail irregularities.

We also estimate that deviations of telescope wheels from circular shape of the amplitude $<0.05 \mathrm{~mm}$ can effectively limit the pointing accuracy to $<0.25 \mathrm{mdeg}$, which is small when compared to other effects investigated in this work. The upper limit of the deviations of the wheels from circular shapes indicates that the most rapidly changing pointing corrections associated with the rail should be stable over time even if the wheels slip.

The pointing corrections when processed through "Model 5" show no evident systematic effects in residuals, suggesting that at the current noise level the model accounts for all major effects that contribute to pointing errors. However, we note that the dispersion of zenith distance corrections is larger than it is in the case of azimuth corrections, which may caused by instabilities of the suspension of the secondary mirror or thermal effects.

The data used for fitting the pointing model were acquired within a relatively short period of time - about 3.5 months of the summer time. While this is may be enough to fit a model, any systematic long term effects (associated with e.g. seasonal temperature variations), if present, may be missed. However, we have shown that if thermal or time dependent effects are under control, the telescope should be capable of pointing with $<12^{\prime \prime}$ accuracy.

Acknowledgments BL would like to thank Kaz Borkowski for his valuable comments on the manuscript and for his previous works on 32-m Torun radio telescope pointing models. BL also would like to thank Jacek Król, Wojtek Szymański, Jacek Jopczyński, Janusz Filarecki, Gieniu Pazderski and Paweł Wolak 
for the maintenance of the telescope secondary mirror and the initial measurements of wheels and the rail. Also, thank you to Marcin Gawroński for help in finding suitable position calibrators, to Marian Szymczak for comments and suggestions, and to Boud Roukema for reading the manuscript. BL wishes to thank anonymous referee for stimulating comments that helped improving the manuscript and gaining new insights.

We acknowledge use of the 'matplotlib' plotting library [14].

This work is based in part on observations carried out using the 32-meter radio telescope operated by Torun Centre for Astronomy of Nicolaus Copernicus University in Torun (Poland) and supported by the Polish Ministry of Science and Higher Education SpUB grant.

Open Access This article is distributed under the terms of the Creative Commons Attribution 4.0 International License (http://creativecommons.org/licenses/by/4.0/), which permits unrestricted use, distribution, and reproduction in any medium, provided you give appropriate credit to the original author(s) and the source, provide a link to the Creative Commons license, and indicate if changes were made.

\section{Appendix: Model of pointing corrections due to rail height irregularities}

In this section we calculate the azimuth and zenith distance pointing corrections due to telescope tilting caused by a local rail indent. We use a simple geometrical model, previously considered by Kaz Borkowski. We assume that the four trolleys of the telescope are connected by a rigid square frame (Section 8.1). The telescope weight split between the trolleys is, in general, not even and may depend on the dish elevation. For example, counter-weights heavier than the dish will cause larger loading of back trolleys with respect to the front ones. When one of the heavily loaded trolleys rolls down an indented region of the rail, the telescope zenith axis will tilt towards that trolley, thus altering the source apparent zenith distance and azimuth. In Fig. 12,

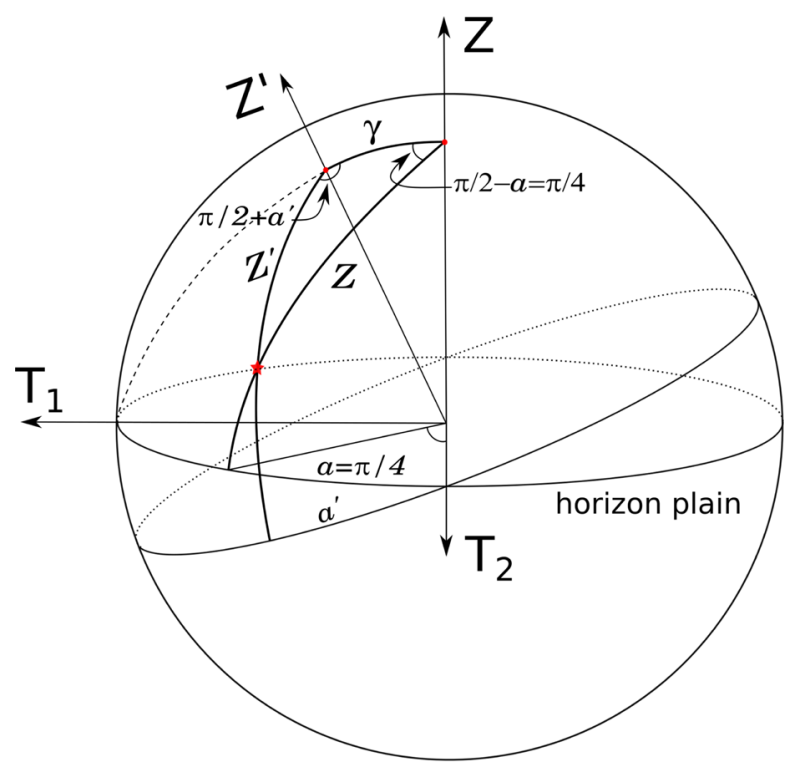

Fig. 12 A model of the effects of rail indent on telescope structure tilt (see text for comments) 
the $\mathrm{Z}$ axis represents the geodetic zenith of a perfect telescope, whereas $\mathrm{Z}$ ' represents a zenith tilted by an angle $\gamma$ towards the trolley located on axis $T_{1}$. The points where axes $\mathrm{Z}$ and $\mathrm{Z}$ ' cross the celestial sphere are marked with red dots. The axis in the horizon plain, $T_{2}$, which is perpendicular to $T_{1}$ is the rotation axis. Without indents, these two axes co-rotate with the telescope in azimuthal motion. The relative azimuth, $a$, of the telescope pointed at a source (marked with a star) is calculated from axis $\mathrm{T}_{2}$ and, by construction, is always equal to $\pi / 4$ (or $\pi / 4+\pi$ depending on which side the telescope zenith is tilted). The source is found at the zenith distance $Z$ from axis $Z$, and at the zenith distance $Z^{\prime}$ from axis $Z^{\prime}$.

The spherical triangle with vertices at the geodetic zenith $(Z)$, the tilted zenith (Z') and at the source (Fig. 12) yields:

$$
\begin{aligned}
\sin Z^{\prime} \sin \left(\frac{\pi}{2}+a^{\prime}\right)= & \sin Z \sin \frac{\pi}{4} \\
\sin Z^{\prime} \cos \left(\frac{\pi}{2}+a^{\prime}\right)= & \sin \gamma \cos Z \\
& -\cos \gamma \sin Z \cos \frac{\pi}{4} \\
\cos Z^{\prime}= & \cos \gamma \cos Z \\
& +\sin \gamma \sin Z \cos \frac{\pi}{4},
\end{aligned}
$$

and it is straightforward to calculate the expected pointing corrections in azimuth and zenith distance:

$$
\begin{aligned}
\tan a^{\prime}-\tan a= & \cos \gamma-1-\sqrt{2} \sin \gamma \cot Z \\
\cos Z^{\prime}-\cos Z= & (\cos \gamma-1) \cos Z \\
& +\frac{\sqrt{2}}{2} \sin \gamma \sin Z,
\end{aligned}
$$

which in the limit of small angles become:

$$
\begin{aligned}
\Delta A \sin (Z) & =\left(a-a^{\prime}\right) \sin (Z) \approx \gamma \cos Z / \sqrt{2} \\
\Delta Z & =Z-Z^{\prime} \approx \gamma / \sqrt{2} .
\end{aligned}
$$

\section{References}

1. Baars, J.W.M., Hooghoudt, B.G., Greve, A., Penalver, J.: Thermal control of the IRAM 30-m millimeter radio telescope. A\&A 195, 364-371 (1988)

2. Bayley, A.M., Davis, R.J., Haggis, J.S., Karcher, H.: Thermal effects on the pointing of the 32-m MERLIN radio telescope at Cambridge. A\&A 283, 1051 (1994)

3. Bolli, P., Orlati, A., Stringhetti, L., Orfei, A., Righini, S., Ambrosini, R., Bartolini, M., Bortolotti, C., Buffa, F., Buttu, M., Cattani, A., D’Amico, N., Deiana, G., Fara, A., Fiocchi, F., Gaudiomonte, F., Maccaferri, A., Mariotti, S., Marongiu, P., Melis, A., Migoni, C., Morsiani, M., Nanni, M., Nasyr, F., Pellizzoni, A., Pisanu, T., Poloni, M., Poppi, S., Porceddu, I., Prandoni, I., Roda, J., Roma, M., Scalambra, A., Serra, G., Trois, A., Valente, G., Vargiu, G.P., Zacchiroli, G.: Sardinia radio telescope. J Astronom Instrum 4, 1550008-880 (2015). https://doi.org/10.1142/S2251171715500087

4. Borkowski K. M.: Rt32 pointing model. Tech. rep., TRAO, Centre for Astronomy, NCU, Torun, Poland. Available at http://www.astro.umk.pl/ kb/Telescopes/Pointing/PointingModel.htm (2004) 
5. Borkowski K. M.: Towards new pointing tables for $32 \mathrm{~m}$ radio telescope. Tech. rep., TRAO, Centre for Astronomy, NCU, Torun, Poland. Available at http://www.astro.umk.pl/ kb/Reports/PointingModel4/ Model4a.htm (2006)

6. Browne, I.W., Mao, S., Wilkinson, P.N., Kus, A.J., Marecki, A., Birkinshaw, M.: OCRA: A one-centimeter receiver array. In: Butcher, H.R. (ed.) Radio Telescopes, Society of Photo-Optical Instrumentation Engineers (SPIE) Conference Series, vol. 4015, pp. 299-307 (2000)

7. Charlot, P., Campbell, R., Alef, W., Borkowski, K., Conway, J., Foley, A., Garrington, S., Kraus, A., Nothnagel, A., Sovers, O., Trigilio, C., Venturi, T., Xinyong, H.: ITRF2000 positions of non-geodetic telescopes in the European VLBI network. In: Behrend, D., Rius, A. (eds.) 15th Workshop Meeting on European VLBI for Geodesy and Astrometry, p. 194 (2001)

8. Chen, Z.P., Kong, D.Q., Li, C.G., Zhu, N.J., Shi, H.L.: Mechanical analysis and measurement of wheel-rail contact system in large aperture radio telescope. In: Society of Photo-Optical Instrumentation Engineers (SPIE) Conference Series, Proc. SPIE, vol. 9906, p. $99064 B$ (2016). https://doi.org/10.1117/12.2232213

9. Gawroński, M.P., Peel, M.W., Lancaster, K., Battye, R.A., Birkinshaw, M., Browne, I.W.A., Davies, M.L., Davis, R.J., Feiler, R., Franzen, T.M.O., Génova-Santos, R., Kus, A.J., Lowe, S.R., Pazderska, B.M., Pazderski, E., Pooley, G.G., Roukema, B.F., Waldram, E.M., Wilkinson, P.N.: 30 GHz observations of sources in the very small array fields. MNRAS 406, 1853-1864 (2010). https://doi.org/10. 1111/j.1365-2966.2010.16784.x

10. Gawronski, W., Baher, F., Gama, E.: Track level compensation look-up table improves antenna pointing precision. In: Society of Photo-Optical Instrumentation Engineers (SPIE) Conference Series, Proc. SPIE, vol. 6273, p. 627316 (2006). https://doi.org/10.1117/12.661259

11. Gawronski, W., Baher, F., Quintero, O.: Azimuth-track level compensation to reduce blind-pointing errors of the deep space network antennas. IEEE Antennas Propag. Mag. 42, 28-38 (2000). https://doi.org/10.1109/74.842123

12. Greve, A., Bremer, M.: Calculated thermal behavior of ventilated high precision radio telescopes. IEEE Antennas Propag. Mag. 48, 9-19 (2006). https://doi.org/10.1109/MAP.2006.277150

13. Himwich W. E.: Pointing model derivation. Tech. rep., NASA/Goddard Space Flight Center. Available at ftp://gemini.gsfc.nasa.gov/pub/fsdocs/model.pdf (1993)

14. Hunter, J.D.: Matplotlib: A 2d graphics environment. Comput. Sci. Eng. 9(3), 90-95 (2007)

15. Kaercher, H.J.: Azimuth axis design for huge telescopes: An update. In: Antebi, J., Lemke, D. (eds.) Astronomical Structures and Mechanisms Technology, Proc. SPIE, vol. 5495, pp. 67-76 (2004). https://doi.org/10.1117/12.553287

16. Kaplan, G., Bartlett, J.L., Monet, A., Bangert, J., Puatua, W., Harris, W., Fredericks, A., Barron, E.G., Barrett, P.: NOVAS: Naval Observatory Vector Astrometry Software Astrophysics Source Code Library (2012)

17. Kong, D.Q., Wang, S.G., Wang, J.Q., Wang, M., Zhang, H.B.: A new calibration model for pointing a radio telescope that considers nonlinear errors in the azimuth axis. Res. Astron. Astrophys. 14, 733-740 (2014). https://doi.org/10.1088/1674-4527/14/6/011

18. Lancaster, K., Birkinshaw, M., Gawroński, M.P., Battye, R., Browne, I., Davis, R., Giles, P., Feiler, R., Kus, A., Lew, B., Lowe, S., Maughan, B., Alareedh, A., Pazderska, B., Pazderski, E., Peel, M., Roukema, B.F., Wilkinson, P.: Sunyaev-Zel'dovich observations of a statistically complete sample of galaxy clusters with OCRA-p. MNRAS 418, 1441-1451 (2011). https://doi.org/10.1111/j.1365-2966. 2011.19241.x

19. Lew, B., Birkinshaw, M., Wilkinson, P., Kus, A.: Simulations of cm-wavelength Sunyaev-Zel'dovich galaxy cluster and point source blind sky surveys and predictions for the RT32/OCRA-f and the Hevelius 100-m radio telescope. J. Cosmol. Astropart. Phys. 2, 004 (2015). https://doi.org/10.1088/ 1475-7516/2015/02/004

20. Lew, B., Roukema, B.F.: The comptonization parameter from simulations of single-frequency, singledish, dual-beam, cm-wave observations of galaxy clusters and mitigating CMB confusion using the Planck sky survey. J. Cosmol. Astropart. Phys. 11, 049 (2016). https://doi.org/10.1088/1475-7516/ 2016/11/049

21. Lew, B., Uscka-Kowalkowska, J.: Clear sky atmosphere at $\mathrm{cm}$-wavelengths from climatology data. MNRAS 455, 2901-2917 (2016). https://doi.org/10.1093/mnras/stv2552

22. Li, N., Li, P., Wu, J., Duan, B.Y.: Modeling the rail surface unevenness of a high-precision radio telescope. Res. Astron. Astrophys. 17, 23 (2017). https://doi.org/10.1088/1674-4527/17/3/23 
23. Lowe, S.R., Gawroński, M.P., Wilkinson, P.N., Kus, A.J., Browne, I.W.A., Pazderski, E., Feiler, R., Kettle, D.: $30 \mathrm{GHz}$ flux density measurements of the Caltech-Jodrell flat-spectrum sources with OCRA-p. A\&A 474, 1093-1100 (2007). https://doi.org/10.1051/0004-6361:20078034

24. Orfei, A., Morsiani, M., Zacchiroli, G., Maccaferri, G., Roda, J., Fiocchi, F.: Active surface system for the new Sardinia Radiotelescope. In: Antebi, J., Lemke, D. (eds.) Astronomical Structures and Mechanisms Technology, Proc. SPIE, vol. 5495, pp. 116-125 (2004). https://doi.org/10.1117/12.548944

25. Peel, M.W., Gawroński, M.P., Battye, R.A., Birkinshaw, M., Browne, I.W.A., Davis, R.J., Feiler, R., Kus, A.J., Lancaster, K., Lowe, S.R., Pazderska, B.M., Pazderski, E., Roukema, B.F., Wilkinson, P.N.: One centimetre receiver array-prototype observations of the CRATES sources at $30 \mathrm{GHz}$. MNRAS 410, 2690-2697 (2011). https://doi.org/10.1111/j.1365-2966.2010.17640.x

26. Pisanu, T., Buffa, F., Morsiani, M., Pernechele, C., Poppi, S.: Thermal behavior of the Medicina 32-meter radio telescope. In: Modern Technologies in Space- and Ground-based Telescopes and Instrumentation, Proc. SPIE, vol. 7739, p. 773935 (2010). https://doi.org/10.1117/12.857422

27. Prestage, R.M., Constantikes, K.T., Balser, D.S., Condon, J.J.: The GBT precision telescope control system. In: Oschmann, J. M. Jr. (ed.) Ground-based Telescopes, Proc. SPIE, vol. 5489, pp. 1029-1040 (2004). https://doi.org/10.1117/12.552319

28. Ries, P., Hunter, T.R., Constantikes, K.T., Brandt, J.J., Ghigo, F.D., Mason, B.S., Prestage, R.M., Ray, J., Schwab, F.R.: Measuring and correcting wind-induced pointing errors of the green bank telescope using an optical quadrant detector. PASP 123, 682 (2011). https://doi.org/10.1086/660677

29. Ries, P.A.: High frequency observations of Iapetus on the Green Bank Telescope aided by improvements in understanding the telescope response to wind. University of Virginia, Ph.D. thesis (2012)

30. Shi, H.L., Chen, Z.P., Wang, S.G., Kong, D.Q., Zhang, J.Y., Zhang, Y.L.: Analysis on wheel-rail contact and rail cracks of $50 \mathrm{~m}$ radio telescope. In: New Technologies for Engineering Research and Design in Industry, Advanced Materials Research, vol. 971, pp. 974-978. Trans Tech Publications, (2014). https://doi.org/10.4028/www.scientific.net/AMR.971-973.974

31. Sun, J.X., Zuo, Y.X., Yang, J., Ma, J.M., Lu, D.R.: Thermal deformation of the Delingha $13.7 \mathrm{~m}$ telescope. Acta Astronomica Sinica 55, 246-255 (2014)

32. Szymczak, M., Olech, M., Wolak, P., Bartkiewicz, A., Gawroński, M.: Discovery of periodic and alternating flares of the methanol and water masers in G107.298+5.639. MNRAS 459, L56-L60 (2016). https://doi.org/10.1093/mnrasl/slw044

33. Ukita, N.: Thermal effects on the pointing of the Nobeyama 45-m telescope. Publ. Nat. Astronom. Observ. Japan 5, 139-147 (1999)

34. Ukita, N.: Wind induced surface deformation of the Nobeyama 45-m radio telescope. In: Groundbased and Airborne Telescopes II, Proc. SPIE, vol. 7012, p. $70120 \mathrm{~V}$ (2008). https://doi.org/10.1117/ 12.787910

35. Ukita, N., Ezawa, H., Ikenoue, B., Saito, M.: Thermal and wind effects on the azimuth axis tilt of the ASTE 10-m antenna. Publ. Nat. Astronom. Observ. Japan 10, 25-33 (2007)

36. von Hoerner, S.: Design of large steerable antennas. AJ 72, 35 (1967). https://doi.org/10.1086/110198

37. Wallace, P.T.: The SLALIB Library. In: Crabtree, D.R., Hanisch, R.J., Barnes, J. (eds.) Astronomical Data Analysis Software and Systems III, Astronomical Society of the Pacific Conference Series, vol. 61, p. 481 (1994) 